BULL. AUSTRAL. MATH, SOC.

\title{
PROPER EMBEDDABILITY \\ OF INVERSE SEMIGROUPS
}

\author{
A. SHEHADAH
}

Let $S$ be an inverse semigroup. We prove that there is a ring with a proper involution $*$ in which $S$ is *-embeddable. The ring will be a natural one, $R[S]$, the semigroup ring of $S$ over any formally complex ring $R$; for example $\mathbb{R}, C$.

\section{Introduction}

In [5] we gave a negative answer to a question in [3]: Given a semigroup $S$ with a proper involution, does there exist a ring $R$ with a proper involution in which $S$ is *-embeddable. In this paper we answer the same question in the affimative if $S$ happens to be an inverse semigroup with an involution equal to its inverse map.

\section{Preliminaries}

DEFINITIONS: Let $S$ be a semigroup. An involution on $S$ is a map * $: S \rightarrow S$ such that, for $a, b \in S, a^{\star \star}=a,(a b) *=b^{\star} a^{\star}$. The involution * is proper if, for $a, b \in S, a a^{*}=a b^{*}=b b^{*}$ implies $a=b$. A proper *-semigroup $(S, *)$ is a ${ }^{*}$-semigroup $(S, \star)$ in which * is proper.

Let $R$ be a unital ring. An involution on $R$ is a map $\star: R \rightarrow R$ such that, for $A, B \in R, A^{* *}=A,(A B)^{\star}=B^{*} A^{*}$, and $(A+B) *=A^{*}+B^{*}$.

Received 2 January 1986. This article is a part of my Ph.D thesis [4], I would like to thank Professor M. Drazin, my advisor. Copyright Clearance Centre, Inc. Serial-fee code: 0004-9727/86 $\$ A 2.00+0.00$. 
The involution * is proper if, for $A \in R, A A^{*}=0$ implies $A=0$. A *-ring $(R, *)$ is a ring $R$ with an involution *. A proper *-ring is a *-ring $\left(R,{ }^{*}\right)$ in which * is proper.

Let $(S, *)$ be a *-semigroup and $(R, *)$ be a *-ring. A *-embedding of $\left(S,{ }^{*}\right)$ into $\left(R,{ }^{*}\right)$ is a $1-1$ map $f: S \rightarrow R$, such that, for $a, b \in S$, $f(a b)=f(a) f(b)$, and $f\left(a^{*}\right)=(f(a))^{*}$. If such a map exists then we say that $(S, *)$ is *-embeddable in $(R, *)$.

Let $(R, *)$ be a *-ring. If for every $A_{1}, \ldots, A_{n} \in R, \sum_{i=1}^{n} A_{i} A_{i}^{*}=0$ implies all $A_{i}=0$, then $\left(R,{ }^{*}\right)$ will be called a formally complex ring. A formally complex ring is a proper *-ring: We take $n=1$ in the definition.

Given a *-semigroup $(S, *)$ and a *-ring $(R, *)$, the *-semigroup ring $\left(R[S],{ }^{*}\right)$ with involution * defined by $\left(\sum r_{i} s_{i}\right)^{*}=\Sigma r_{i}^{*} s_{i}^{*}$ is a *-ring in which $(S, *)$ is *-embeddable. If $(S, *)$ is a proper *-semigroup, there may not exist a proper *-ring $\left(R,{ }^{*}\right)$ in which $(S, *)$ is *-embeddable [5].

\section{The results}

LEMMA. Let $S$ be an inverse semigroup and let $a, b, c$ be three eleinents of $S$ such that $a a^{-1}=b c^{-1}$. Then $a^{-1} b=a^{-1} c$.

Proof. We have $a a^{-1}=b c^{-1}=a b^{-1}$. Therefore $b b^{-1} \cdot a a^{-1}$ $=b b^{-1} \cdot b c^{-1}=b b^{-1} b \cdot c^{-1}=b c^{-1}=a a^{-1}$. Thus $b b^{-1} a a^{-1} a=a a^{-1} a$; therefore, $b b^{-1} a=a$. Now we have $a^{-1} b\left(a^{-1} b\right)^{-1}=a^{-1} b \cdot b^{-1} a=a^{-1} \cdot b b^{-1} a$ $=a^{-1} a$, and $a^{-1} b\left(a^{-1} c\right)^{-1}=a^{-1} b \cdot c^{-1} a=a^{-1} \cdot b c^{-1} \cdot a=a^{-1} \cdot a a^{-1} \cdot a=a^{-1} a$. Thus $a^{-1} b \leq a^{-1} c$ in the vagner - Preston partial ordering. similarly $a^{-1} c \leq a^{-1} b$ and so $a^{-I_{b}}=a^{-1} c$.

THEOREM. Let $S$ be an inverse semigroup and $(R, *)$ be any formally complex ring. Then the involution * induced on the semigroup ring $R[S]$ is proper. 
Proof. We have to show, for every $m$, for every finite subset $\left\{s_{1}, \ldots, s_{m}\right\} \subseteq S$ and for every $A=\sum_{i=1}^{m} a_{i} a_{i} \in R[S]$, that $A A^{*}=0$ only if $a_{i}=0(i=1, \ldots, m)$. We prove this by complete induction on $m$. The case $m=1$ is trivial, since here $a a \cdot a^{\star} s^{-1}=a a^{\star} . s s^{-1}$, and so $(a s) .(a s)^{*}=0$ only if $a a^{*}=0$ so that $a=0$ (since $R$ is a proper *-ring).

Given any positive integer $n$, assume the result is true for all $m \leq n$. Choose any subset $\left\{s_{1}, \ldots, s_{n}\right\} \subseteq S$, and let $a_{1}, \ldots, a_{n} \in R$ be such that $A=\sum_{i=1}^{n} a_{i} \varepsilon_{i}$ satisfies $A A^{*}=0$. Pick from the set $\left\{s_{1}, \ldots, s_{n}\right\}$ any $s_{j}$ maximal with respect to the vagner - Preston order, without loss of generality assume it is $s_{n}$, that is, $s_{n} \leq s_{i}$ $(i=1, \ldots, n-1)$. We distinguish the following two cases, which exhaust all other possibilities:

Case 1. ${ }^{6} \varepsilon^{8} n^{-1}=\varepsilon_{u} s_{v}^{-1}$ implies $u=v(\forall u, v=1, \ldots, n)$. Case 2. ${ }^{s} s_{n}^{-1}=s_{u} s_{v}^{-1}$ for some pair $(u, v)$ such that $u \neq v$. We treat each case separately.

In Case 1 the only $s_{u} v^{-1}$ which are equal to $s_{n} s^{-1}$ are of the form $s_{i} s_{i}^{-1}$. Without loss of generality, let these be $s_{k} s_{k}^{-1} \ldots, s_{n} s_{n}^{-1}$ for some $k$ in the range $1 \leq k \leq n$. Then by collecting the coefficients of $s_{n} s^{-1}$ in $A A^{*}$, we have that $a_{k} a_{k}+\ldots+a_{n} a_{n}^{\star}=0$. Hence, since $R$ is formally complex, $a_{k}=\ldots=a_{n}=0$. Thus $A=\sum_{i=1}^{k-1} a_{i} s_{i}$.

In Case 2, apart from $s_{n} s_{n}^{-1}$ itself, there can be no $s_{n} s^{-1}$ or ${ }^{8} v_{n}^{s^{-1}}$ equal to $\varepsilon_{n} \varepsilon_{n}^{-1}$ since either would imply $\varepsilon_{n} \leq \theta_{v}$ contrary to our choice of $s_{n}$. Also $s_{n}^{-1} s_{n} \neq s_{n}^{-1} s_{v}$ for otherwise $s_{n}=\varepsilon_{v}$.

$$
\text { Now } \varepsilon_{n}^{-1} A\left(\varepsilon_{n}^{-1} A\right) \star=\varepsilon_{n}^{-1} A A^{*} s_{n}=0 \text { where } \varepsilon_{n}^{-1} A={ }_{i=1}^{n} a_{i} s_{n}^{-1} \varepsilon_{i} \text {. }
$$


Also $s_{n} s_{n}^{-1}=\varepsilon_{u} s^{-1}$ for at least one pair $(u, v)$ with $u, v, n$ all different, and for all such $(u, v)$ we have $s_{n}^{-1} s_{u}=s_{n}^{-1} s_{v}$, by the Lemma. Thus, in the formal sum $s_{n}^{-1} A=\sum_{i=1}^{n} a_{i} s_{n}^{-1} s_{i}$, there is at least one "collapsing" $s_{n}^{-1} s_{u}=s_{n}^{-1} s_{v} \neq s_{n}^{-1} s_{n}$ with $u, v, n$ all different. In other words $s_{n}^{-1} A$ can be written in the form $s_{n}^{-1} A=b_{n} s_{n}^{-1} s_{n}+b_{2} s_{2}^{\prime}+\ldots+b_{i} s_{i}^{\prime}$ for some $s_{2}^{\prime}, \ldots, s_{i}^{\prime} \in S$, where $b_{n}=a_{n}, b_{2}, \ldots, b_{i}+R$ and $i<n$. Since $s_{n}^{-1} A\left(s_{n}^{-1} A\right) \star=0$, by the induction hypothesis, $a_{n}=b_{n}=0$. Thus $A={ }_{i=1}^{n-1} a_{i} s_{i}$. We have shown that, in both Case 1 and Case $2, A$ is a formal sum with fewer than $n$ terms. By the induction hypothesis it follows that all $a_{i}=0$.

PROPOSITION. Let $S$ be any inverse semigroup and let $R$ be any formally complex *-ring. Then the semigroup ring $R[S]$ contains no nonzero nil ideal. (Equivalently, $R[S]$ has a zero nil radical).

PROOF. The map $A=\Sigma r_{i} s_{i} \rightarrow A^{*}=\sum r_{i}^{*} s_{i}^{-1}$ defines an involution on $R[S]$ - From the Theorem, $R[S]$ is a proper *-ring. Let $I$ be any nil ideal in $R[S]$ and let $A \in I$. Now $A A^{*} \in I$ and hence, for some $n \geq 1,\left(A A^{*}\right)^{n}=0$. By *-cancellation [2] $A=0$. Thus $I=0$.

\section{References}

[1] A. Clifford and G. Preston, The Algebraic Theory of Semigroups, Math. Surveys, Amer. Math. Soc., Providence, R.I. 7 (1969).

[2] M. Drazin, "Regular Semigroups with Involution", Symposizon on Regular Semigroups, Northern Illinois University (1979), 29-48.

[3] M. Drazin, "Natural Structures on Rings and Semigroups with Involution", (To appear).

[4] A. Shehadah, Embedding Theorems for Semigroups with Involution, Ph.D Thesis, Puxdue University, West Lafayette, Indiana, (1982). 
[5] A. Shehadah, "A Counter Example on *-embeddability into Proper *-rings", (To appear).

Department of Mathematics

Yarmouk University

Irbid

Jordan 